\title{
1 Psilocybin rescues sociability deficits in an animal model of autism
}

3 Irene Mollinedo-Gajate ${ }^{1}$, Chenchen Song ${ }^{1}$, Marcos Sintes-Rodriguez ${ }^{1}$, Tobias Whelan

$4 \quad 2$, Anaïs Soula², Aslihan Selimbeyoglu², Shaun Hurley², Thomas Knöpfel ${ }^{1 *}$

5

$6{ }^{1}$ Laboratory for Neuronal Circuit Dynamics, Department of Brain Sciences, Imperial 7 College London, London, UK

$8 \quad{ }^{2}$ COMPASS Pathways Ltd., London, UK 9

10 Corresponding authors: Irene Mollinedo-Gajate, and Thomas Knöpfel. Laboratory for 11 Neuronal Circuit Dynamics, Department of Brain Sciences, Imperial College London, 12 Du Cane Road, Burlington Danes Building, London, W12 ONN, UK.

13 Emails: i.mollinedo-gajate@imperial.ac.uk; t.knopfel@imperial.ac.uk.

\section{Abstract}

19 Autism spectrum disorder (ASD) is characterized by core deficits in social interaction.

20 The classic serotonergic psychedelic psilocybin has been suggested as a therapeutic agent that may ameliorate in the core symptomology of ASD. We found that the acute response to psilocybin was attenuated in the prenatal valproic acid exposure mouse model of ASD, and importantly, psilocybin rescued the social behavioural abnormalities present in these ASD model mice. 
Autism spectrum disorder (ASD) is a heterogenous neurodevelopmental disorder characterized by core deficits in social interaction and communication, repetitive or restricted behaviour and interests, and altered sensory sensitivity ${ }^{1}$. The aetiology and pathophysiology of ASD remains largely unknown, and is considered to be multifactorial, encompassing both genetic and environmental factors ${ }^{2-4}$. The lack of a single molecular mechanism underlying the aetiology of ASD has contributed to the challenge of finding efficacious therapeutic interventions.

The regulation and functionality of the serotonergic (5-HT) system has been implicated to play an important role in the core pathophysiology of $A S D^{5-9}$, hence its modulation is an evidence-based therapeutic target ${ }^{10-12}$. Psilocybin is produced by several species of mushrooms, and is one of the compounds responsible for the psychedelic effects that these mushrooms induce, likely via altering $5-\mathrm{HT}$ signalling through $5-\mathrm{HT}_{2 \mathrm{~A}}$ receptors ${ }^{13,14}$. Reduced cortical 5- $\mathrm{HT}_{2 \mathrm{~A}}$ receptor expression has been reported in ASD suggesting that this population may be less sensitive to the subjective effects of psilocybin 8,9 . Psychedelics have recently shown potential safety and efficacy for alleviating various neuropsychiatric disorders ${ }^{15}$. Recent human studies also showed that psilocybin can produce sustained prosocial behaviour ${ }^{16,17}$, and affects several facets of social cognition relevant to deficits in $A S D^{18,19}$.

47 Preclinical evaluation of psilocybin's potential to ameliorate altered sociability requires 48 a suitable animal model that recapitulates aspects of the social deficits observed in ASD. One such model, based on prenatal exposure to valproic acid (VPA), is particularly attractive as it does not bias towards a single genetic alteration as in genetic models of ASD and thus may better represent idiopathic ASD. VPA is clinically used for the treatment of epileptic seizures, bipolar mania, and as a migraine prophylactic. Through this long-standing clinical use, it has been observed that pregnant mothers treated with VPA are at an increased risk of giving birth to children that are diagnosed with $\mathrm{ASD}^{20}$. In mice, prenatal VPA exposure also results in offspring exhibiting many ASD-like behavioural phenotypes ${ }^{21-24}$, including altered social behaviour ${ }^{25-27}$. Here we explored the potential of psilocybin to ameliorate altered sociability in the prenatal VPA mouse model of autism. Offspring that have been exposed to VPA in utero are hereon referred to as VPA group, and those exposed to 
60

61

62

63

64

65

66

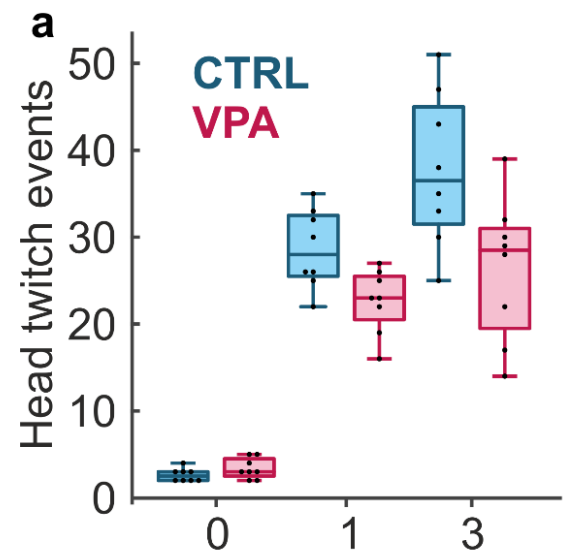

Psilocybin dose $(\mathrm{mg} / \mathrm{kg})$ see Extended Data Fig. 2, Supplementary Table. 1.

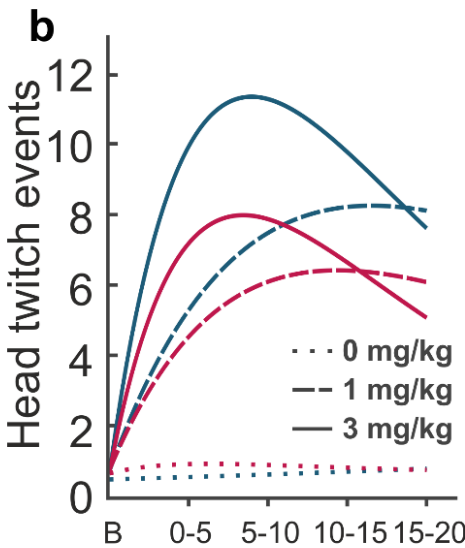

Time ( $\min )$

Fig. 1: VPA group shows reduced head twitch response induced by psilocybin.

a, Total count of head twitch events over $20 \mathrm{~min}$, induced by an intraperitoneal injection of saline or psilocybin in Control and VPA groups. Psilocybin evoked dose-dependent HTR in both groups. HTR count was significantly lower with no significant dosedependency in VPA mice $\left(F_{\text {pre-treatment } x \text { treatment(2,42) }}=4.895, P=0.012\right)$. Boxplot: center line, median; box limits, upper and lower quartiles; whiskers, 1.5x interquartile range. b, Fitted time course of head twitch events above baseline (B) in 5-min time bins. VPA and Control groups showed similar HTR time course shapes. $n=8 /$ group. For detail 
86

24-hour after psilocybin/saline treatments, animals were tested for their social behaviour (sociability and social memory), to capture effects beyond the acute action of psilocybin as well as to avoid acute psychedelic-like effects confounding social behaviour. VPA group spent significantly less time engaging in social interaction compared to Control group, as quantified by the total duration of nose-to-nose interactions with the stranger mouse (VPAsaL vs CTRLsaL: $t=3.282, P=0.005$; Fig. 2a). A low dose of psilocybin (1 mg/kg i.p.) restored the sociability behaviour of VPA group to a level similar to that of Control group (VPAsAL vs VPAPS11: $t=2.928, P=$ 0.009; Fig. 2a), but the rescue effect of psilocybin was not evident following the higher dose ( $3 \mathrm{mg} / \mathrm{kg}$; VPAsal vs VPApsiz: $t=1.071, P=0.300$; Fig. 2a). Surprisingly, psilocybin treatment in the Control group showed a dose-dependent trend in reducing the total nose-to-nose interaction duration, although this effect was not statistically significant (CTRLsal vs CTRLPSI1: $t=0.657, P=0.523$; CTRLsaL vs CTRLPSI3: $t=$ 1.799, $P=0.953$; Fig. 2a). The reduced total nose-to-nose interaction duration in VPA group was due to reduced average duration of individual interactions (VPAsAL vs CTRLsal: $t=2.952, P=0.010) .1 \mathrm{mg} / \mathrm{kg}$ psilocybin substantially restored this component of social interaction behaviour and increased the duration of individual interactions when compared with the VPA saline group (VPAsaL Vs VPAPS11: $t=3.307$, $P=0.004$; Fig. 2b). However, we did not observe any difference in the average time per interaction between VPA mice treated with $3 \mathrm{mg} / \mathrm{kg}$ psilocybin versus saline controls (VPAsAL vs VPAPSI3: $t=1.326, P=0.203$ ). The surprising trend (though statistically non-significant) of psilocybin-induced reduction in social interaction in Control group was also observed in a dose-dependent trend in the average duration of individual interactions in both high and low dose psilocybin treated control mice when compared to the control saline group (CTRLsAL vs CTRLPS11: $t=1.698, P=0.115$; CTRLsaL vs CTRLpsI3: $t=1.911, P=0.078$ ).

Next, we assessed the effect of psilocybin on social novelty preference by introducing a new stranger mouse (S2) in addition to the presence of the existing stranger mouse (S1). The social novelty index derived from this measure quantifies the extent to which $\mathrm{S} 2$ is recognized as new. As this recognition required remembering $\mathrm{S} 1$, this index is also a measure of short-term social memory. The social novelty index was reduced for the VPA group, and a single treatment of psilocybin (at either low or high doses) in VPA group increased their social novelty preference to a level similar (higher) than 
119 that of the Control group (Fig. 2c, Supplementary Table. 1). The opposite effect was observed in the Control group treated with either $1 \mathrm{mg} / \mathrm{kg}$ or $3 \mathrm{mg} / \mathrm{kg}$ psilocybin, where

a

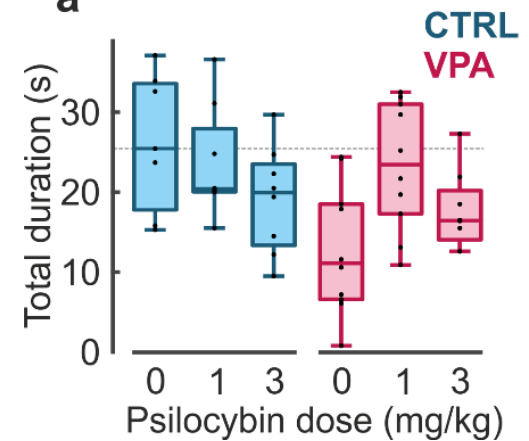

b

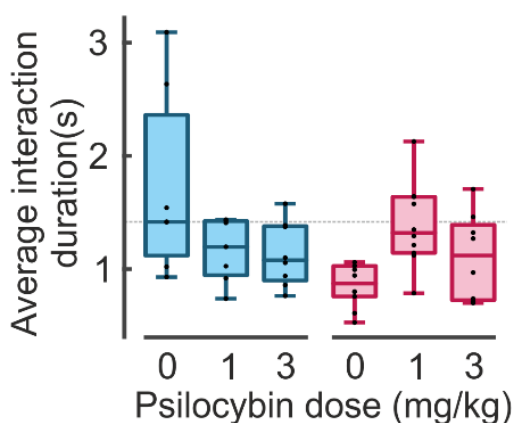

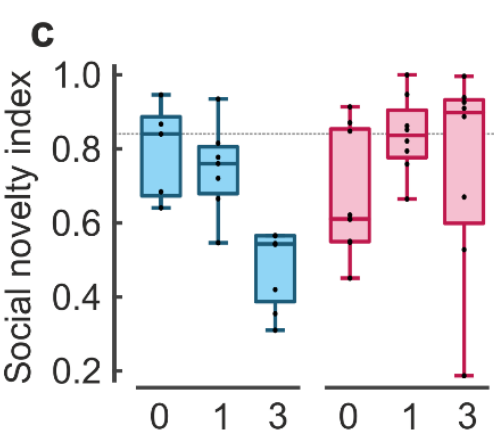

Psilocybin dose $(\mathrm{mg} / \mathrm{kg})$

Fig. 2: Single administration of psilocybin improves sociability and social

memory in ASD model mice. a, Total duration time of direct nose to nose interactions

during 10-min observation period ( $\left.F_{\text {pre-treatment } x \text { treatment(2,45) }}=3.633, P=0.034\right)$, 24 hours following a single administration of saline or psilocybin in Control and VPA groups. $\mathbf{b}$,

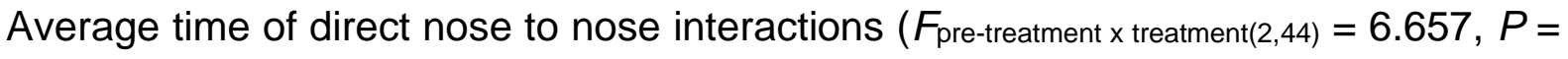
0.003), related to observations in a. c, Social novelty index is dose-dependently reduced in the Control group 24 hours following a single administration of saline or

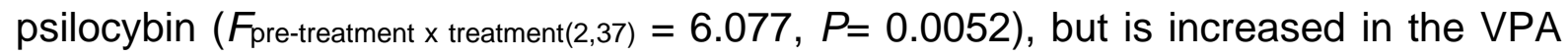
group. Two-way ANOVA: $n=7$ control saline, $n=7$ control PSI $1, n=8$ control PSI 3 , $n=10$ VPA saline, $n=10$ VPA PSI $1, n=8$ VPA PSI 3. Dotted line represents median value from the Control group administered with saline. Boxplot: center line, median; box limits, upper and lower quartiles; whiskers, 1.5x interquartile range; outliers not shown. For statistics see Supplementary Table. 1

Psychedelic research is currently undergoing a renaissance, with the therapeutic potential of these compounds being increasingly recognised. Psilocybin, for example, has recently received "breakthrough designation" by the FDA in treatment-resistant depression $^{29}$ and major depressive disorder ${ }^{30}$. Towards a reverse translational approach, here we report the efficacy of psilocybin in an animal model of ASD as evidence that psilocybin may be a promising pharmacotherapy for altered sociability in ASD. More detailed mechanistic studies will be needed in future to understand the molecular and circuit underpinnings of this therapeutic potential. 


\section{Methods}

148 Animals

149 All experimental procedures were performed at Imperial College London UK in 150 accordance with the United Kingdom Animal Scientific Procedures Act (1986), under 151 Home Office Personal and Project Licences following appropriate ethical review. Time152 mated C57BL/6J mice (Charles River, UK) were housed in groups, and separated two 153 days prior to the expected littering date. Offspring were weaned at P28 and housed in 154 groups of up to five animals per cage after weaning. All animals were maintained in 155 ventilated cages, on a $12 / 12 \mathrm{~h}$ light/dark cycle at $21 \pm 2^{\circ} \mathrm{C}$ and $55 \pm 10 \%$ humidity. Water and food were provided ad libitum. Eight weeks old mice of both genders were used for behavioural experiments. All experiments were conducted during the daytime.

\section{Prenatal VPA treatment}

160 Valproic acid (2-propylpentanoic acid) sodium salt was obtained from Sigma Aldrich (P4543; Germany), and freshly dissolved in sterile saline $(0.9 \% \mathrm{NaCl})$ to $50 \mathrm{mg} / \mathrm{ml}, \mathrm{pH}$ 7.4. On embryonic day 10.5 (E10.5), pregnant females were intraperitoneally administered with either a single dose of $500 \mathrm{mg} / \mathrm{kg}$ VPA or equal volume of saline. The dose and the embryonic day of VPA administration were based on previously published reports ${ }^{21-23}$. Prenatally exposed VPA animals showed significantly lower body weight than the prenatal saline control at weaning age (controls $=15.01 \mathrm{~g}$, VPA $=12.09$ g; $P=0.0002 ; n=24$ group; Extended Data Fig. 1, Supplementary Table. 1) and remained lower at week 8 (controls $=20.95 \mathrm{~g}, \mathrm{VPA}=18.40 \mathrm{~g} ; P=0.009 ; \mathrm{n}=$ abnormalities were observed in the prenatal VPA animals: 60\% showed kinked tail, $10 \%$ had moderate facial abnormalities, $6 \%$ displayed fur problems, $3 \%$ had misaligned teeth. These abnormalities were not observed in the control group.

\section{Drug administration}

175 Psilocybin ([3-(2-dimethylaminoethyl)-1H-indol-4-yl] dihydrogen phosphate, Onyx 176 Scientific Ltd, UK) was dissolved in sterile saline solution $(0.9 \% \mathrm{NaCl})$ to appropriate 177 concentrations.

178 For behavioural experiments, 8-week old offspring were intraperitoneally injected 179 either with saline or psilocybin (1 mg/kg, PSI 1; or $3 \mathrm{mg} / \mathrm{kg}, \mathrm{PSI} 3$ ) 24 hours prior to 
180

181

182

183

184

185

186

187

188

189

190

191

192

193

194

195

196

197

198

199

200

201

202

203

204

205

206

207

208

209

210

211

212

the sociability and social memory test. The treatments led to six different test groups: control saline, control psilocybin $1 \mathrm{mg} / \mathrm{kg}$, control psilocybin $3 \mathrm{mg} / \mathrm{kg}$, VPA saline, VPA psilocybin $1 \mathrm{mg} / \mathrm{kg}$, VPA psilocybin $3 \mathrm{mg} / \mathrm{kg}$.

\section{Head twitch response}

Head twitch events were evaluated immediately after the i.p. administration of either saline or psilocybin for a period of 20 minutes. The mouse was placed into a customized behavioural box immediately after injection and was free to explore. The number of head-twitch events were counted by direct observation in 5-minute bins. The box $(25.5 \times 12.5 \times 12.5 \mathrm{~cm}[\mathrm{~L} \times \mathrm{W} \times \mathrm{H}])$ was made of red Plexiglass and the floor was covered with clean sawdust.

\section{Sociability and social memory behavioural test}

Sociability and social novelty preference experiments were performed in a custombuilt transparent red Plexiglass box with matt white acrylic floor. The total arena $(60 \mathrm{x}$ $40 \times 22 \mathrm{~cm}$ [ $\mathrm{L} \times \mathrm{W} \times \mathrm{H}]$ ) was divided in three smaller evenly sized chambers ( $20 \times 40$ $x 22 \mathrm{~cm}$ [ $\mathrm{L} \times \mathrm{W} \times \mathrm{H}]$ ) interconnected by $4 \times 4 \mathrm{~cm}$ doors cut out of both central walls. Clear Plexiglass cylinders $(10.5 \mathrm{~cm}$ internal diameter, $11 \mathrm{~cm}$ external diameter, $16 \mathrm{~cm}$ length) with $1-\mathrm{cm}$ slits and $0.5-\mathrm{cm}$ rods were used as cups. A red acrylic lid was placed on top of both cups to prevent animals from escaping or falling. The box was placed in a dark and quite room, illuminated from above with infrared LEDs located 1 meter over the arena.

Each behavioural testing experiment took place over 4 consecutive days (Extended

Data Fig. 1). Animals were acclimatised to the testing room for at least one hour before the start of experiments on each day. Each test mouse was placed into the central chamber and allowed to freely explore the three-chamber setup for 10 minutes over 3 consecutive days. During this pre-habituation time, doors to the side chambers were kept open and empty cups were placed into the two side chambers at similar positions as the final test day (day 4). Cup mice were individually pre-habituated to the cups also for 10 minutes over 3 consecutive days. On day 4 , the three-chamber test was performed in three phases (Habituation, Sociability, Social Memory). Each test animal was allowed to freely explore all three chambers for 10 minutes with empty cups (Habituation Phase), then driven to the central chamber with the doors closed while 
213 an age-, size-, and gender-matched unfamiliar mouse (stranger 1) was being placed

214 into one of the cups. The test mouse was then allowed to freely explore chambers for 21510 minutes (Sociability Phase). The test mouse was then returned to the central 216 chamber while a second unfamiliar mouse (stranger 2) was placed in the remaining 217 empty cup. Finally, the test mouse was allowed to move freely across the three 218 chambers for 10 minutes (Social Memory Phase).

219 All behavioural sessions were video-recorded using a CMOS camera (Basler 220 acA2000-165umNIR) and Basler software (Basler AG, Germany). Video recordings were used to track the position of the body of the animal in each chamber, as well as to score the duration time of direct nose to nose interactions and interaction events by a blinded well-trained observer.

224

\section{Data analysis and statistics}

226 No statistical methods were used to predetermine sample size, but our sample size was selected based on standards in the field. Experiments were blinded to VPA or control prenatal treatment, as well as treatment during automatically or manually analysis. If applicable, outliers were identified and excluded using a Grubb's test. Differences between two groups were compared using unpaired two-tailed Student's $t$-test when data were normally distributed, otherwise the Mann-Whitney $U$ test was used. Multiple group differences were assessed using two- and three-way analysis of variance (ANOVA). Independent variables were defined as: pre-treatment (Control or VPA), treatment (PSI1: psilocybin $1 \mathrm{mg} / \mathrm{kg}$, or PSI3: psilocybin $3 \mathrm{mg} / \mathrm{kg}$, or $\mathrm{SAL}$ : saline), gender. Multiple comparisons were further assessed by Bonferroni's post hoc test. Definition of statistical significance was set at an alpha value of 0.05 . Specific $P$ values are reported for each analysis in the corresponding figure legend and Supplementary Table 1. All statistical analyses were performed using GraphPad Prism 8 (San Diego, CA, USA) and InVivoStat (Cambridge, United Kingdom).

Social novelty index was calculated as the following:

interaction duration with S2

244 HTR responses were fitted using MATLAB (R2019b) with a single dose 245 pharmacokinetics curve with the following equation: 


$$
\mathrm{HTR}=\frac{\mathrm{F} \cdot \mathrm{k}_{\mathrm{a}}}{\left(\mathrm{k}_{\mathrm{a}}-\mathrm{k}_{\mathrm{e}}\right)} \cdot\left(\mathrm{e}^{-\mathrm{k}_{\mathrm{e}} \cdot \mathrm{t}}-\mathrm{e}^{-\mathrm{k}_{\mathrm{a}} \cdot \mathrm{t}}\right)+\mathrm{B}
$$

247 Where: HTR = head twitch response; $\mathrm{F}=$ drug factor (dose and bioavailability); $\mathrm{k}_{\mathrm{a}}=$

248 absorption rate constant, $\mathrm{k}_{\mathrm{e}}=$ elimination rate constant; $\mathrm{t}=$ time; $\mathrm{B}=$ baseline count. 
a

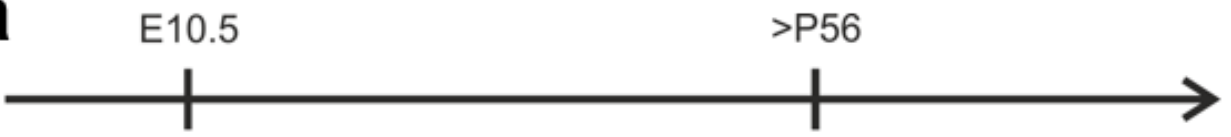

Prenatal treatment ip injection in dam VPA - $500 \mathrm{mg} / \mathrm{kg}$ CTRL - saline
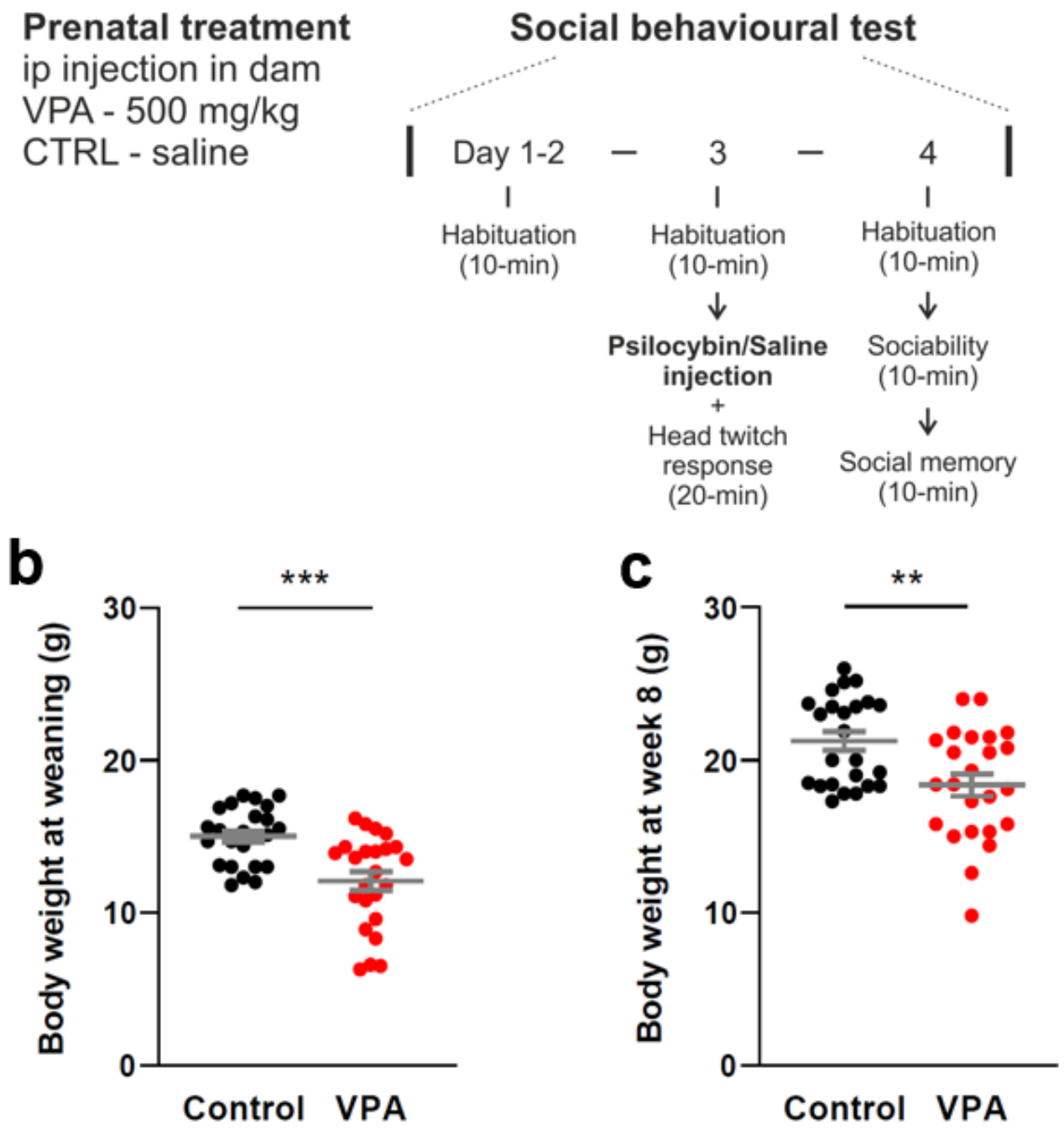

Extended Data Fig. 1: Experimental timeline and VPA model. a, Experimental 282 timeline. Pregnant mothers were intraperitoneally injected with saline or $500 \mathrm{mg} / \mathrm{kg}$ 283 VPA at E10.5. 8-week old offspring were pre-habituated to the three-chamber arena 284 for three consecutive days. After pre-habituation on day 3, saline or psilocybin was 285 intraperitoneally injected, and the head twitch response (HTR) was counted. On day 2864 , animals were tested for sociability and social memory using the three-chamber test. 287 b, c, Mice from VPA group had lower body weight than the Control group at weaning 288 age (b, $t=4.033, P<0.001, \mathrm{n}=24 /$ group) and at 8-week old (c, $U=163.5, P=0.009$, $289 \mathrm{n}=24$ /group). Mean \pm SEM. ${ }^{* *} P<0.01,{ }^{* \star *} P<0.001$. 

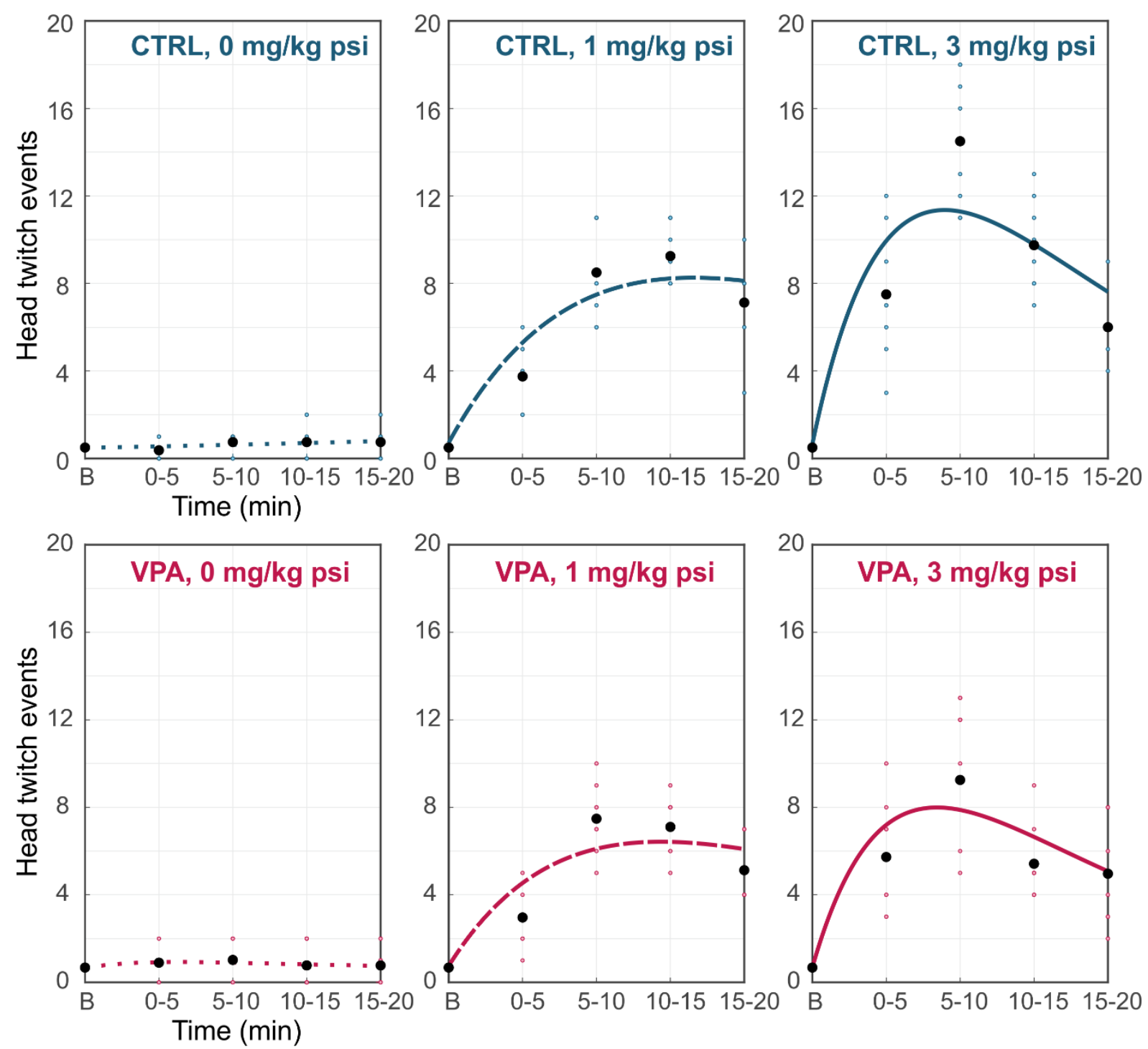

Extended Data Fig. 2: Fitted time course of head twitch events above baseline

(B) in 5 min time bins for individual groups. Head twitch events observed in 5-min bins over a total duration of 20-min was fitted with a single dose pharmacokinetics equation. Individual datapoints (blue/pink dots for Control/VPA groups respectively) and group mean values (black dots) are shown. $\mathrm{N}=8 /$ group. 


\section{Acknowledgements}

303 We thank Roxanne Wood, Bonnie Glen, and Chantelle Day for their assistance with

304 animal husbandry and care, Imperial Hackspace for their assistance with the 305 behavioural set-up construction, and all members of the Knöpfel lab for discussions. 306 This work was funded by a collaboration grant from COMPASS Pathways, and funding 307 from EU H2020 MSCA 813986 Syn2Psy ITN.

308

\section{Author contributions}

310 I.M-G., M.S-R., and C.S. performed the experiments and analysed the data. I.M-G., 311 T.K., T.W., and C.S. conceived the project and wrote the paper. A.Soula, A. 312 Selimbeyoglu and S.H. contributed to the study design.

314 Competing interests

315 T.W., A. Soula, A. Selimbeyoglu, and S.H. are employees of COMPASS Pathways 316 Ltd. COMPASS Pathways Ltd. had no influence over the execution or publication of 317 this study.

\section{Data and code availability}

320 The data and MATLAB scripts that support the findings of this study are available upon 321 reasonable request from the corresponding author. 


\section{References}

1 American Psychiatric Association. in Diagnostic and Statistical Manual of Mental Disorders (Washington, DC, 2013).

2 Betancur, C. Etiological heterogeneity in autism spectrum disorders: more than 100 genetic and genomic disorders and still counting. Brain Res 1380, 42-77, doi:10.1016/j.brainres.2010.11.078 (2011).

3 Bolte, S., Girdler, S. \& Marschik, P. B. The contribution of environmental exposure to the etiology of autism spectrum disorder. Cell Mol Life Sci 76, 1275-1297, doi:10.1007/s00018-018-2988-4 (2019).

4 Chaste, P. \& Leboyer, M. Autism risk factors: genes, environment, and geneenvironment interactions. Dialogues Clin Neurosci 14, 281-292 (2012).

5 Muller, C. L., Anacker, A. M. J. \& Veenstra-VanderWeele, J. The serotonin system in autism spectrum disorder: From biomarker to animal models. Neuroscience 321, 2441, doi:10.1016/j.neuroscience.2015.11.010 (2016).

6 Veenstra-VanderWeele, J. \& Blakely, R. D. Networking in autism: leveraging genetic, biomarker and model system findings in the search for new treatments. Neuropsychopharmacology 37, 196-212, doi:10.1038/npp.2011.185 (2012).

7 Oblak, A., Gibbs, T. T. \& Blatt, G. J. Reduced serotonin receptor subtypes in a limbic and a neocortical region in autism. Autism Res 6, 571-583, doi:10.1002/aur.1317 (2013).

8 Beversdorf, D. Q. et al. 5-HT2 receptor distribution shown by [18F] setoperone PET in high-functioning autistic adults. J Neuropsychiatry Clin Neurosci 24, 191-197, doi:10.1176/appi.neuropsych.11080202 (2012).

9 Murphy, D. G. et al. Cortical serotonin 5-HT2A receptor binding and social communication in adults with Asperger's syndrome: an in vivo SPECT study. Am J Psychiatry 163, 934-936, doi:10.1176/ajp.2006.163.5.934 (2006).

10 Reddihough, D. S. et al. Effect of Fluoxetine on Obsessive-Compulsive Behaviors in Children and Adolescents With Autism Spectrum Disorders: A Randomized Clinical Trial. JAMA 322, 1561-1569, doi:10.1001/jama.2019.14685 (2019).

11 Gould, G. G. et al. Density and function of central serotonin (5-HT) transporters, 5$\mathrm{HT} 1 \mathrm{~A}$ and 5-HT2A receptors, and effects of their targeting on BTBR $\mathrm{T}+\mathrm{tf} / \mathrm{J}$ mouse social behavior. J Neurochem 116, 291-303, doi:10.1111/j.1471-4159.2010.07104.x (2011).

12 Arakawa, H. Somatosensorimotor and Odor Modification, Along with Serotonergic Processes Underlying the Social Deficits in BTBR T+ Itpr3(tf)/J and BALB/cJ Mouse Models of Autism. Neuroscience, doi:10.1016/j.neuroscience.2020.02.002 (2020).

13 Madsen, M. K. et al. Psychedelic effects of psilocybin correlate with serotonin 2A receptor occupancy and plasma psilocin levels. Neuropsychopharmacology 44, 13281334, doi:10.1038/s41386-019-0324-9 (2019).

14 Vollenweider, F. X., Vollenweider-Scherpenhuyzen, M. F., Babler, A., Vogel, H. \& Hell, D. Psilocybin induces schizophrenia-like psychosis in humans via a serotonin-2 agonist action. Neuroreport 9, 3897-3902, doi:10.1097/00001756-199812010-00024 (1998).

15 Rucker, J. J. H., lliff, J. \& Nutt, D. J. Psychiatry \& the psychedelic drugs. Past, present \& future. Neuropharmacology 142, 200-218, doi:10.1016/j.neuropharm.2017.12.040 (2018).

16 Forstmann, M., Yudkin, D. A., Prosser, A. M. B., Heller, S. M. \& Crockett, M. J. Transformative experience and social connectedness mediate the mood-enhancing effects of psychedelic use in naturalistic settings. Proc Natl Acad Sci U S A 117, 23382346, doi:10.1073/pnas.1918477117 (2020).

17 Smigielski, L., Scheidegger, M., Kometer, M. \& Vollenweider, F. X. Psilocybin-assisted mindfulness training modulates self-consciousness and brain default mode network connectivity with lasting effects. Neuroimage 196, 207-215, doi:10.1016/j.neuroimage.2019.04.009 (2019). 
18 Pokorny, T., Preller, K. H., Kometer, M., Dziobek, I. \& Vollenweider, F. X. Effect of Psilocybin on Empathy and Moral Decision-Making. Int $J$ Neuropsychopharmacol 20, 747-757, doi:10.1093/ijnp/pyx047 (2017).

19 Preller, K. H. et al. Effects of serotonin $2 \mathrm{~A} / 1 \mathrm{~A}$ receptor stimulation on social exclusion processing. Proc Natl Acad Sci U S A 113, 5119-5124, doi:10.1073/pnas.1524187113 (2016).

20 Christensen, J. et al. Prenatal valproate exposure and risk of autism spectrum disorders and childhood autism. JAMA 309, 1696-1703, doi:10.1001/jama.2013.2270 (2013).

21 Brumback, A. C. et al. Identifying specific prefrontal neurons that contribute to autismassociated abnormalities in physiology and social behavior. Mol Psychiatry 23, 20782089, doi:10.1038/mp.2017.213 (2018).

22 Wang, R. et al. Aberrant Development and Synaptic Transmission of Cerebellar Cortex in a VPA Induced Mouse Autism Model. Front Cell Neurosci 12, 500, doi:10.3389/fncel.2018.00500 (2018).

23 Gogolla, N. et al. Common circuit defect of excitatory-inhibitory balance in mouse models of autism. J Neurodev Disord 1, 172-181, doi:10.1007/s11689-009-9023-x (2009).

24 Gandal, M. J. et al. Validating gamma oscillations and delayed auditory responses as translational biomarkers of autism. Biol Psychiatry 68, 1100-1106, doi:10.1016/j.biopsych.2010.09.031 (2010).

25 Nicolini, C. \& Fahnestock, M. The valproic acid-induced rodent model of autism. Exp Neurol 299, 217-227, doi:10.1016/j.expneurol.2017.04.017 (2018).

26 Sakade, Y., Yamanaka, K., Soumiya, H., Furukawa, S. \& Fukumitsu, H. Exposure to valproic acid during middle to late-stage corticogenesis induces learning and social behavioral abnormalities with attention deficit/hyperactivity in adult mice. Biomed Res 40, 179-188, doi:10.2220/biomedres.40.179 (2019).

27 Campolongo, M. et al. Sociability deficits after prenatal exposure to valproic acid are rescued by early social enrichment. Mol Autism 9, 36, doi:10.1186/s13229-018-02219 (2018).

28 Gonzalez-Maeso, J. et al. Hallucinogens recruit specific cortical 5-HT(2A) receptormediated signaling pathways to affect behavior. Neuron 53, 439-452, doi:10.1016/j.neuron.2007.01.008 (2007).

29 COMPASS Pathways receives FDA Breakthrough Therapy designation for psilocybin therapy for treatment-resistant depression, <https://compasspathways.com/compasspathways-receives-fda-breakthrough-therapy-designation-for-psilocybin-therapy-fortreatment-resistant-depression/>

30 FDA grants Breakthrough Therapy Designation to Usona Institute's psilocybin program for major depressive disorder, $<$ https://www.businesswire.com/news/home/20191122005452/en/FDA-grants- 